\title{
Clinical impact and cost-effectiveness of a 176-condition expanded carrier screen
}

\author{
Kyle A. Beauchamp, PhD (10 ${ }^{1}$, Katherine A. Johansen Taber, $\mathrm{PhD}^{1}$ and Dale Muzzey, $\mathrm{PhD}^{1}$
}

Purpose: Carrier screening identifies couples at high risk for conceiving offspring affected with serious heritable conditions. Minimal guidelines recommend offering testing for cystic fibrosis and spinal muscular atrophy, but expanded carrier screening (ECS) assesses hundreds of conditions simultaneously. Although medical societies consider ECS an acceptable practice, the health economics of ECS remain incompletely characterized.

Methods: Preconception screening was modeled using a decision tree comparing minimal screening and a 176-condition ECS panel. Carrier rates from $>60,000$ patients, primarily with private insurance, informed disease incidence estimates, while cost and life-years-lost data were aggregated from the literature and a costof-care database. Model robustness was evaluated using one-way and probabilistic sensitivity analyses.

Results: For every 100,000 pregnancies, 290 are predicted to be affected by ECS-panel conditions, which, on average, increase mortality by 26 undiscounted life-years and individually incur $\$ 1,100,000$ in lifetime costs. Relative to minimal screening, preconception ECS reduces the affected birth rate and is estimated to be cost-effective (i.e., $<\$ 50,000$ incremental cost per life-year), findings robust to perturbation.

Conclusion: Based on screened patients predominantly with private coverage, preconception ECS is predicted to reduce the burden of Mendelian disease in a cost-effective manner compared with minimal screening. The data and framework herein may facilitate similar assessments in other cohorts.

Genetics in Medicine (2019) 21:1948-1957; https://doi.org/10.1038/s41436019-0455-8

Keywords: cost-effectiveness; expanded carrier screening

\section{INTRODUCTION}

Collectively, Mendelian diseases are a significant cause of infant mortality and hospitalization. ${ }^{1,2}$ The American College of Obstetricians and Gynecologists (ACOG), ${ }^{3,4}$ the American College of Medical Genetics and Genomics (ACMG), ${ }^{5}$ and other US medical societies recommend offering carrier screening for select prevalent conditions. ACOG recommends offering cystic fibrosis (CF) and spinal muscular atrophy (SMA) screening for all women considering pregnancy (or already pregnant), ${ }^{4}$ as well as additional screening based on family history and ethnicity.

Expanded carrier screening (ECS), often performed using next-generation sequencing (NGS), screens tens to hundreds of conditions. ${ }^{6,7}$ ACOG has recognized ECS as an acceptable screening strategy, ${ }^{3}$ and the American Medical Association Current Procedural Terminology (CPT) Editorial Panel has approved a CPT code (81443, effective January 2019) for panethnic sequencing-based ECS panels with $\geq 15$ genes. ${ }^{8}$ With these recent actions, now is an opportune time to evaluate the impact of ECS on the health system.
Cost-effectiveness analysis ${ }^{7,9-11}$ evaluates the cost and clinical benefits of medical interventions. It condenses each comparison between interventions into a single number (incremental costeffectiveness ratio; ICER) that summarizes the cost (e.g., dollars) per unit of health benefit outcome (e.g., life-years gained). This framework allows thoughtful comparisons of interventions that maximize patient health while optimally allocating society's finite health-care resources. Applying standard costeffectiveness principles to preconception screening presents challenges when modeling net health outcomes and costs. One common modeling choice, used herein, leverages a hypothetical wherein births of sick children are replaced by births of healthy children as a result of screening.

Though examined for individual conditions, ${ }^{12-15}$ the costeffectiveness of ECS is not fully characterized. One study found NGS-based ECS with a 14-condition panel to be costeffective. ${ }^{7}$ Others, though not directly addressing ECS, examined the cost-effectiveness of treatments: due to high prices, recently approved drugs treating ECS conditions (e.g., $\mathrm{CF}^{16}$ and $\mathrm{SMA}^{17}$ ) may not be cost-effective.

${ }^{1}$ Myriad Women’s Health (formerly Counsyl), South San Francisco, CA, USA. Correspondence: Kyle A. Beauchamp (mwh_research@myriad.com) or

Dale Muzzey (mwh_research@myriad.com)

Submitted 16 July 2018; accepted: 24 January 2019

Published online: 14 Fubruary 2019 
Here we model the clinical impact and cost-effectiveness of preconception ECS, building upon previous work ${ }^{7}$ in several ways. First, we consider a large panel with 176 conditions that is currently used for routine screening. Second, we aggregate disease cost and life-years-lost data from both literature and cost-of-care databases. Third, we model disease incidence using a cohort of more than 60,000 patients. Finally, we use a decision model-guided by survey results from couples who underwent preconception ECS testing and primarily carried private insurance-to compare the cost-effectiveness of ECS relative to a minimal screening protocol or no screening.

\section{MATERIALS AND METHODS}

\section{Decision tree}

A decision tree model was chosen as the most parsimonious protocol that captures the key workflows of preconception carrier screening (Fig. 1). First, couples may be at risk or not at risk for a panel condition. Second, we assume that the detection rate for each condition is $100 \%$, so that couples deemed not at risk have zero chance of having a child affected with a screened condition (see "Discussion"). Third, at-risk couples (ARCs; defined below) have a fixed probability of choosing a reproductive intervention to avert any possibility of a child affected by a screened condition; this probability $(180 / 235, \sim 77 \%)$ is estimated from a recent clinical utility study. ${ }^{18}$ This estimate of the preconception ARC intervention rate was chosen over estimates from previous studies ${ }^{19,20}$ because of its large sample size (235 responding ARCs) and because it specifically included preconception ARCs who were at risk for at least 1 of the 176 conditions examined herein. Because any single disease may have been undersampled in the survey study, we assume the aggregated intervention rate applies for all diseases on the panel (the panel is composed almost entirely of conditions with severe or profound impact $^{21}$ ). As this rate could be skewed by various factors (e.g., a population favorably predisposed toward genetic
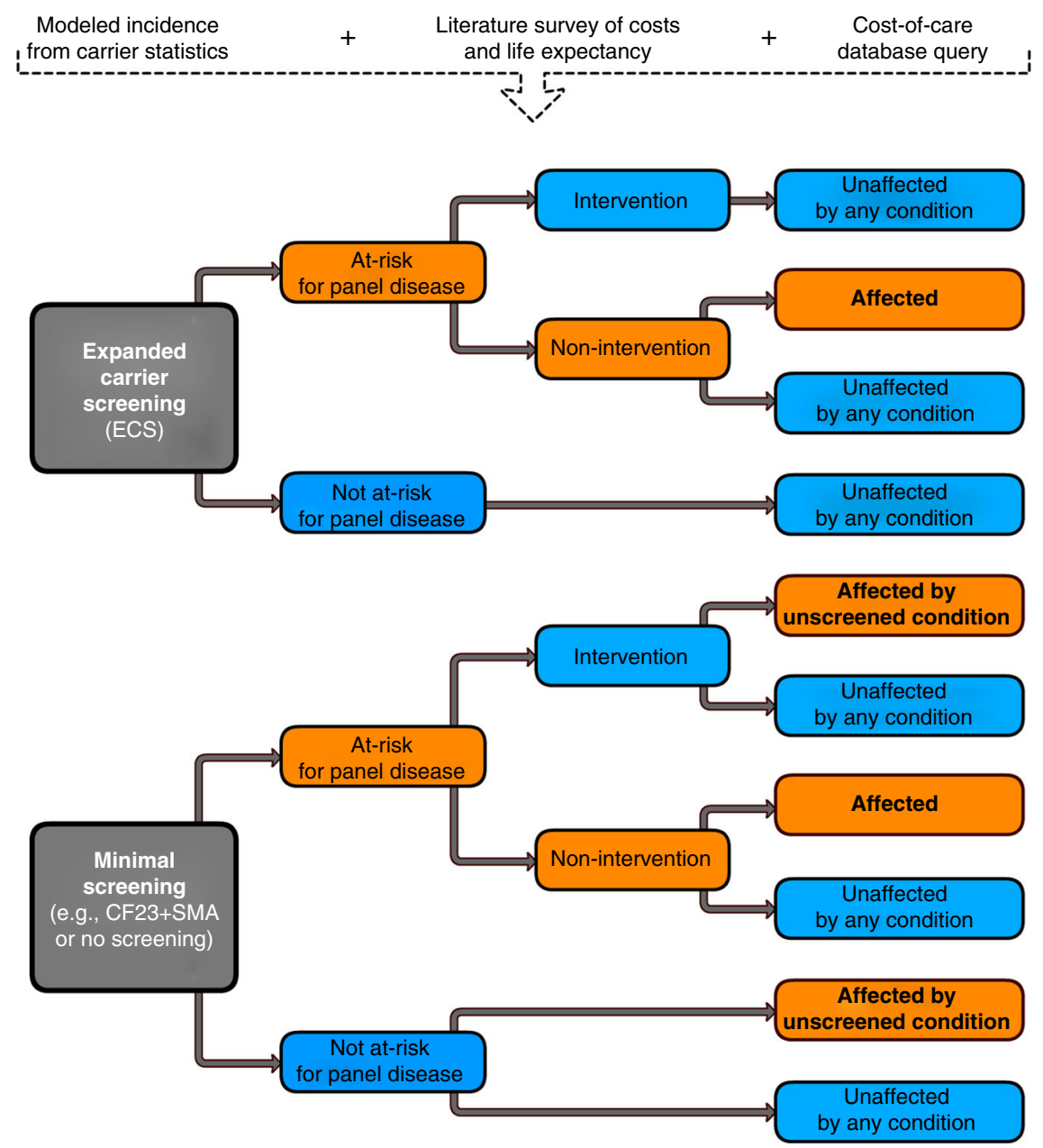

Fig. 1 Decision tree model. Top: Analyses in this work combined three data sources to construct a decision tree model. Bottom: The decision tree model considered in this work - one arm for expanded carrier screening (ECS) and another for minimal screening (cystic fibrosis and spinal muscular atrophy [CF23 + SMA] screening or no screening)—depicts the risk status, intervention choice (where applicable), and pregnancy outcome from left to right. Riskmitigating and unaffected nodes are blue, whereas risk-increasing, risk-maintaining, and affected nodes are orange. The decision tree, like the rest of the modeling described herein, assumes that ECS tests for the totality of diseases; therefore, "Unaffected by any condition" means that the pregnancy is not affected for a condition screened on the ECS panel, and "Affected by unscreened condition" means a pregnancy is affected by a condition on the ECS panel beyond CF23 + SMA. 
testing), we evaluated a broad range of intervention rates (see "Results").

Although many screening workflows are possible, here we model a tandem workflow, in which both partners are tested concurrently on the same sequencing-based ECS platform. This choice automatically accounts for partner testing costs by pricing tests per couple. Although the model does not specifically account for genetic counseling utilization, genetic counseling is provided at no additional charge to all patients who receive ECS at Myriad Women's Health (MWH) and is thus implicitly included in cost and clinical outcome metrics herein.

\section{Cost-effectiveness perspective}

For the economic evaluations herein, we typically assume the analytical perspective of private, commercial payers, and therefore focus on costs on 1- and 3-year time horizons. However, we also report a number of quantities (e.g., lifetime costs, life-years) that are of interest to other stakeholders. The primary health outcomes of interest are life-years gained, assuming replacement of affected births with unaffected births. Additional health metrics of interest to stakeholders include the number of affected births and the number of reproductive interventions. The interventions of interest herein are either ECS or minimal (e.g., CF23 + SMA) carrier screening, followed by optional intervention to avoid an affected birth (e.g., adoption, in vitro fertilization [IVF], prenatal diagnosis, etc.).

\section{Study population}

This study uses models informed by screening results from a large cohort ( $>60,000$ patients, $>200$ ARCs) to investigate the potential impact of more broadly applying panethnic ECS. Despite the size of this cohort, it does not perfectly reflect the entire US population in terms of the distribution of ethnicity, geography, religion, or socioeconomic status. We attempted to account for ethnicity biases in analysis of disease incidence by reweighting ethnicity-specific ARC rates by the US frequency of each ethnicity (see "Incidence modeling"). However, for the intervention rate we cannot directly account for socioeconomic, geographic, and religious biases (see "Discussion"), though we indirectly explore the impact that biases in these factors could have on our results via sensitivity analysis (described below) and by reporting results for a range of intervention rates. The rate of reproductive interventions pursued by ARCs is estimated from surveys of ARCs from patients who underwent preconception ECS. ${ }^{18}$ Insurance coverage for this survey cohort is skewed toward private payers: approximately $10 \%$ of claims were billed to government-sponsored insurers (i.e., Medicaid, Medicare, or managed care organization [MCO]), underrepresenting the $\sim 50 \%$ of US births covered under Medicaid. ${ }^{22}$

\section{Institutional review board approval}

This study was designated as exempt by Western Institutional Review Board. All patients provided informed consent for testing and anonymized research.

\section{Disease list}

This study examines the 176-condition Foresight ${ }^{\mathrm{tm}}$ Universal Panel $^{23}$ (see Supplementary Table S1; MWH). While the CPT and ACOG definitions of ECS permit panels of different sizes, we studied this panel because it is the presently offered ECS in our laboratory. For this study, we refer to this panel as ECS, but evaluate other panel sizes in Table S7.

\section{Data sources: overview}

Our analyses utilize per-disease estimates of incidences, lifeyears lost, and disease treatment costs, which we collected from three sources (Fig. 1; top). First, aggregated carrier statistics were used to model fetal disease incidences (see "Incidence modeling"). Second, a literature survey of disease cost and life expectancy data was performed (see "Literature survey"). Third, we acquired data on aggregated health outcomes and expenses for a longitudinal cohort of millions of patients (Truven Health Analytics; see "Cost-of-care database survey" and Supplementary Table S4). Supplementary Tables S2 and S3 contain the value and provenance of quantities used in this work.

Disease cost estimates were adjusted to 2018 dollars using the personal consumption expenditures (PCE) health-care price index. ${ }^{24,25}$ When from a literature source, life-years and lifetime cost data were assumed to be present-value discounted appropriately. When such parameters were modeled herein (e.g., from lifespan data; see Supplementary Methods), life-years lost and lifetime costs were present-value adjusted assuming a discount rate of $3 \%\left(\right.$ ref. ${ }^{26}$ ).

\section{Incidence modeling}

Because affected persons for each condition are rare, we modeled disease incidence using the inheritance patterns and carrier frequencies estimated from 66,036 patients screened using the 176-condition universal panel. This modeled fetal disease risk (MFDR) approach, described previously, ${ }^{27,28}$ predicted the frequency of affected conceptuses. To reduce bias, the cohort excluded patients with fertility issues or family history of disease.

To estimate the rate of at-risk couples (ARCs) in which both partners are carriers for an autosomal recessive condition or the mother is a carrier for an X-linked condition, we note that for both autosomal recessive and $\mathrm{X}$-linked conditions, the ratio of ARC rate to MFDR is 4, so we define the ARC rate as $4{ }^{*}$ MFDR. For diseases with complex inheritance (e.g., fragile $\mathrm{X}$ syndrome), this approximation simplifies the decision analysis. Panel-wide MFDR and ARCs were treated as additive across the panel's constituent diseases. ${ }^{28}$ Calculations assumed intraethnicity coupling and were reweighted to match the ethnic makeup of the general US population, ${ }^{29}$ as described previously (Supp. Table S1 in ref. ${ }^{28}$ ). In the case of cystic fibrosis, we excluded nine variants known to have variable clinical presentation or modified inheritance (see Supporting Methods and Table S9). 


\section{Literature survey}

We performed a literature survey to aggregate the following quantities for each condition: lifetime disease cost, years 0-3 (postbirth) disease cost, years 0-1 (postbirth) disease cost, and life-years lost. We included scientific publications, government documents, reports from disease support groups, and clinical resources for patients as acceptable literature. For cost estimates of several FDA-approved orphan drugs (e.g., nusinersen/Spinraza ${ }^{\mathrm{Tx}}$ ), we used FDA indication documents, reports from published news sources (e.g., Forbes), and reports from trade organizations (e.g., America's Health Insurance Plans; AHIP). We primarily used Google Scholar for queries, with search terms based on the disease and/or gene names and keywords including "life expectancy," "lifeyears," "lifespan," "mortality," and "cost." The search did not explicitly filter on time, but recent articles (>1990) were preferred when available.

To estimate the cost of follow-up reproductive care for the ARCs who intervene to avoid an affected birth, we integrated the probabilities and costs of preimplantation genetic testing for monogenic conditions (PGT-M), IVF, prenatal diagnosis (PD), and termination from Table 1 in ref. ${ }^{7}$; each reproductive intervention was estimated to cost $\$ 13,783$, primarily driven by the cost of IVF (see Supporting Methods for details).

For many diseases, life expectancy was found directly in recent literature. In cases without a directly stated life expectancy, we estimated it using the life-years lost among cohorts of affected individuals (see Supporting Methods).

\section{Cost-of-care database survey}

Short-term disease cost data were queried from a commercial database (Marketscan; Truven Health Analytics). ${ }^{30-32}$ Twenty-five conditions were selected based on having high incidence and specific International Classification of Diseases (ICD) coding. For each disease, ICD-9 or ICD-10 codes were chosen to maximize specificity and cohort size (see Table S4).

For each disease, several queries were performed (full output in Supplementary Table S4). In this work, only the first-year cost and three-year cost numbers were used (cells C37 and C39 on each condition's tab in Table S4). To estimate the disease costs during the first $N$ years of life (where $N$ is either 1 or 3 ), we did the following. First, patients were included based on age and continuous enrollment during the appropriate time window. Second, patients were labeled as being diagnosed or not diagnosed based on whether they received an appropriate diagnosis code at some point in the portion of their life chronicled in the database. Finally, for both commercial payers ("com") and Medicaid ("med") separately, the annual average costs of the diagnosis cohort (" $\mu \_d x$ ") and an appropriately matched (based on age, eligibility, time window, payer) nondiagnosis cohort (" $\mu \_$nodx") were calculated. The reported disease cost enforced nonnegativity (disease_cost $>0$ ) and, where possible, prioritized commercial payers (versus Medicaid), as indicated below:

$$
\begin{aligned}
& \text { If } \mu \_d x_{\text {com }}>\mu_{-} n o d x_{\text {com }}: \\
& \quad \text { disease } \_ \text {cost }=\mu_{-} d x_{\text {com }}-\mu_{-} n o d x_{\text {com }} \\
& \text { else if } \mu \_d x_{m e d}>\mu_{-} n o d x_{m e d}: \\
& \quad \text { disease_cost }=\mu_{-} d x_{\text {med }}-\mu_{-} n o d x_{m e d} \\
& \text { else : }
\end{aligned}
$$

disease_cost $=0$

We note that ICD coding may lead to false positives in the diagnosed cohort $^{33}$ and, consequently, disease_cost underestimation.

\section{Missing value estimation}

Quantities with missing data were estimated from the remaining values. For lifetime disease costs, we used the following rules: if available, use a literature estimate of the lifetime cost; otherwise, discount and accumulate the annual cost for each year in the life expectancy; if the annual cost or life expectancy is unknown, use the cost of the first three years of life; if the cost of the first three years of life is unknown, discount and accumulate three years of annual costs. In the case of missing life expectancy data, we truncate disease costs at 3 years with the goal of being conservative. For values unaddressed by these rules, we assigned the value to be the median of other available corresponding values. For example, the unavailable first-year cost for glycogen storage disease type Ia is estimated using the median first-year cost among all diseases for which values are available. Finally, in cases with multiple data points for a single cost parameter, we used the largest cost (due to medical inflation and new treatments, a recent estimate was generally selected).

\section{Screening modes}

We examined screening modes that represent the different theoretical and practical ways in which carrier screening could be adopted and evaluated:

- "No Screening." Couples are not screened for any panel condition.

- "Population Impact: CF23 + SMA." Couples are screened for the 23 ACMG-recommended CF variants ${ }^{34}$ and SMA. All ARCs intervene to avoid an affected birth. Couples may still be at risk for conditions beyond CF23 and SMA.

- "Population Impact: ECS." Couples are screened for 176 conditions. All ARCs intervene to avoid an affected birth.

- "77\% Intervention CF23 + SMA." Couples undergo CF23 + SMA screening, but $77 \%$ (180/235) of ARCs intervene to avoid affected births. ${ }^{18}$

- "77\% Intervention ECS." Couples undergo ECS in which $\sim 77 \%(180 / 235)$ of ARCs intervene to avoid affected births.

- "50\% Intervention CF23 + SMA." Couples undergo CF23 + SMA screening, but $50 \%$ of ARCs intervene to avoid affected births. 
- “50\% Intervention ECS." Couples undergo ECS in which $50 \%$ ARCs intervene to avoid affected births.

The two "Population Impact" models isolate the population impact of panel diseases (ECS or CF23 + SMA) without consideration of the intervention behavior of ARCs. The other screening modes examined both the impact of diseases and the screening interventions that ARCs pursue. Note that the "Population Impact" (i.e., 100\% intervention) scenarios are not meant to be interpreted as realistic interventions, but as a tool to separately assess the impact of disease and the impact of screening. The "77\% Intervention" models were included because the $77 \%$ intervention rate was observed in the largest studied ARC cohort that underwent preconception ECS. ${ }^{18}$ Cost-effectiveness calculations based on this rate could be overly favorable because the survey cohort included patients who elected to undergo genetic screening and may be more inclined toward reproductive interventions than patients in other populations (see "Discussion"). Therefore, we also included a " $50 \%$ Intervention" model to represent populations less inclined toward reproductive interventions.

\section{Sensitivity analysis}

We assessed model sensitivity in two ways: ${ }^{9,10}$ (1) one-way sensitivity analysis, in which all but one variables were held at their base values and the remaining value was set to levels that capture realistic bounds on the variable; and (2) probabilistic sensitivity analysis, whereby all variables were drawn from realistic prior distributions and outcome metrics are assessed across the ensemble of sampled parameter vectors. Model parameters, base values, ranges, and prior distributions are summarized in Supplementary Table S2, and described in the Supplementary Methods.

\section{Software}

Software versions are provided in Supplementary Table S6.

\section{RESULTS}

\section{Disease incidence and cost estimates}

For each disease, we estimated incidence and costs based on screening results from 66,036 patients, a literature survey of $\sim 80$ published resources, and queries from a cost-of-care database; results for the ten most frequent conditions are in Table 1 (full results in Tables S3 and S5). Incidences span multiple orders of magnitude: common diseases like $\beta$-chain hemoglobinopathies, CF, Duchenne/Becker muscular dystrophy, and fragile $\mathrm{X}$ syndrome together affect more than 100 per 100,000 pregnancies, whereas the panel's 150 least common diseases collectively affect approximately 40 per 100,000 pregnancies. Lifetime cost estimates are also highly variable, with several diseases exceeding $\$ 1,000,000$ per affected individual. Early costs are skewed as well, due to some diseases having onset in infancy (e.g., SMA) and others in adolescence/adulthood (e.g., Fabry disease).

\section{Clinical and economic impacts of screening}

We built a decision tree model (see "Materials and methods") to assess the impact of ECS diseases in terms of both clinical outcomes (e.g., number of ARCs, affected births averted, and life-years lost) and economic factors (e.g., spending over first year, first three years, and lifetime) (Table 2).

Modeling predicted that $1.2 \%$ of couples will be at high risk for a screened condition, with 290 in 100,000 births affected (Table 2: "Population Impact"). Due to the high mortality associated with ECS conditions, the per-birth rate of lost lifeyears was 0.016 , or 0.074 without discounting (Table 2). Among couples who underwent preconception ECS and were identified as ARCs, $77 \%$ intervened $^{18,19}$ to avoid an affected birth (via, e.g., IVF with preimplantation genetic testing for monogenic diseases, use of donor gamete, adoption, or decision to avoid pregnancy), meaning that ECS would avert 220 affected births per 100,000 pregnancies (Table 2: "77\% Intervention ECS"). Finally, the model also estimated the

Table 1 Disease frequency and cost analysis

\begin{tabular}{|c|c|c|c|c|c|c|c|}
\hline Disease & $\begin{array}{l}\text { Frequency } \\
(1 \text { in } X)\end{array}$ & $\begin{array}{l}\text { Lifetime } \\
\text { cost }\end{array}$ & $\begin{array}{l}\text { Years } 0-3 \\
\text { cost }\end{array}$ & $\begin{array}{l}\text { Years } \\
0-1 \text { cost }\end{array}$ & $\begin{array}{l}\text { Weighted } \\
\text { lifetime cost }\end{array}$ & $\begin{array}{l}\text { Weighted } \\
\text { years } 0-3 \text { cost }\end{array}$ & $\begin{array}{l}\text { Weighted } \\
\text { years } 0-1 \text { cost }\end{array}$ \\
\hline $\mathrm{Hb} \beta$-chain-related hemoglobinopathies & 2097 & $\$ 528,054$ & $\$ 36,851$ & $\$ 14,857$ & $\$ 252$ & $\$ 18$ & $\$ 7$ \\
\hline Cystic fibrosis & 3242 & $\$ 3,031,311$ & $\$ 94,274$ & $\$ 20,600$ & $\$ 935$ & $\$ 29$ & $\$ 6$ \\
\hline $\begin{array}{l}\text { Dystrophinopathy (including Duchenne/ } \\
\text { Becker muscular dystrophy) }\end{array}$ & 3374 & $\$ 1,165,575$ & $\$ 53,737$ & $\$ 7013$ & $\$ 345$ & $\$ 16$ & $\$ 2$ \\
\hline Phenylalanine hydroxylase deficiency & 8484 & $\$ 153,766$ & $\$ 283,936$ & $\$ 97,542$ & $\$ 18$ & $\$ 33$ & $\$ 11$ \\
\hline Congenital adrenal hyperplasia & 9962 & $\$ 137,826$ & $\$ 70,647$ & $\$ 29,797$ & $\$ 14$ & $\$ 7$ & $\$ 3$ \\
\hline Spinal muscular atrophy & 11,096 & $\$ 2,084,993$ & $\$ 1,172,570$ & $\$ 450,000$ & $\$ 188$ & $\$ 106$ & $\$ 41$ \\
\hline Smith-Lemli-Opitz syndrome & 13,214 & $\$ 205,858$ & $\$ 205,858$ & $\$ 129,020$ & $\$ 16$ & $\$ 16$ & $\$ 10$ \\
\hline
\end{tabular}

US-weighted frequency, cost per affected birth, and cost per unscreened birth weighted by frequency are tabulated for the ten most common diseases. Cost figures are shown for the lifetime, $0-1$ year and $0-3$ year time periods, where year 0 is birth. 


\begin{tabular}{|c|c|c|c|c|c|c|c|c|c|}
\hline Panel & $\begin{array}{l}\text { At-risk } \\
\text { couples } \\
\text { detected }\end{array}$ & $\begin{array}{l}\text { Number of } \\
\text { reproductive } \\
\text { interventions }\end{array}$ & $\begin{array}{l}\text { Affected } \\
\text { births } \\
\text { averted }\end{array}$ & $\begin{array}{l}\text { Life-years } \\
\text { gained }\end{array}$ & $\begin{array}{l}\text { Life-years } \\
\text { gained } \\
\text { undiscounted }\end{array}$ & $\begin{array}{l}\text { Lifetime cost } \\
\text { averted }\end{array}$ & $\begin{array}{l}\text { Three-year cost } \\
\text { averted }\end{array}$ & $\begin{array}{l}\text { Year } 1 \text { cost } \\
\text { averted }\end{array}$ & $\begin{array}{l}\text { Intervention } \\
\text { costs accrued }\end{array}$ \\
\hline $\begin{array}{l}\text { Population Impact: } \\
\text { ECS }\end{array}$ & $1.16 \mathrm{E}-02$ & $1.16 \mathrm{E}-02$ & $2.90 \mathrm{E}-03$ & $1.61 \mathrm{E}-02$ & 7.43E-02 & $\$ 3249.88$ & $\$ 405.66$ & $\$ 130.10$ & $\$ 159.77$ \\
\hline $\begin{array}{l}\text { Population } \\
\text { Impact: CF23 + SMA }\end{array}$ & 1.27E-03 & 1.27E-03 & 3.17E-04 & 4.15E-03 & $1.61 \mathrm{E}-02$ & $\$ 874.74$ & $\$ 127.03$ & $\$ 45.22$ & $\$ 17.46$ \\
\hline $\begin{array}{l}\text { Population Impact: } \\
\text { Difference }\end{array}$ & 1.03E-02 & 1.03E-02 & $2.58 \mathrm{E}-03$ & 1.19E-02 & $5.82 \mathrm{E}-02$ & $\$ 2375.14$ & $\$ 278.63$ & $\$ 84.88$ & $\$ 142.31$ \\
\hline $\begin{array}{l}77 \% \text { Intervention } \\
\text { ECS }\end{array}$ & 1.16E-02 & 8.88E-03 & $2.22 \mathrm{E}-03$ & 1.23E-02 & 5.69E-02 & $\$ 2489.27$ & $\$ 310.72$ & $\$ 99.65$ & $\$ 122.38$ \\
\hline $\begin{array}{l}77 \% \text { Intervention } \\
\text { CF23 + SMA }\end{array}$ & $1.27 \mathrm{E}-03$ & $9.70 \mathrm{E}-04$ & $2.43 \mathrm{E}-04$ & 3.18E-03 & $1.23 \mathrm{E}-02$ & $\$ 670.02$ & $\$ 97.30$ & $\$ 34.64$ & $\$ 13.37$ \\
\hline $\begin{array}{l}\text { 77\% Intervention: } \\
\text { Difference }\end{array}$ & $1.03 \mathrm{E}-02$ & 7.91E-03 & $1.98 \mathrm{E}-03$ & $9.14 \mathrm{E}-03$ & 4.46E-02 & $\$ 1819.25$ & $\$ 213.42$ & $\$ 65.01$ & $\$ 109.00$ \\
\hline $\begin{array}{l}50 \% \text { Intervention } \\
\text { ECS }\end{array}$ & 1.16E-02 & 5.80E-03 & $1.45 \mathrm{E}-03$ & 8.04E-03 & $3.71 \mathrm{E}-02$ & $\$ 1624.94$ & $\$ 202.83$ & $\$ 65.05$ & $\$ 79.89$ \\
\hline $\begin{array}{l}50 \% \text { Intervention } \\
\text { CF23 + SMA }\end{array}$ & $1.27 \mathrm{E}-03$ & $6.33 \mathrm{E}-04$ & $1.58 \mathrm{E}-04$ & 2.07E-03 & 8.04E-03 & $\$ 437.37$ & $\$ 63.52$ & $\$ 22.61$ & $\$ 8.73$ \\
\hline $\begin{array}{l}50 \% \text { Intervention: } \\
\text { Difference }\end{array}$ & $1.03 \mathrm{E}-02$ & $5.16 \mathrm{E}-03$ & $1.29 \mathrm{E}-03$ & 5.96E-03 & 2.91E-02 & $\$ 1187.57$ & $\$ 139.31$ & $\$ 42.44$ & $\$ 71.16$ \\
\hline
\end{tabular}

Difference

Costs exclude ECS screening costs, which are considered separately in the ICER analysis (Fig. 2). All values are reported as per-couple rates.

CF cystic fibrosis, ECS expanded carrier screening, ICER incremental cost-effectiveness ratio, SMA spinal muscular atrophy.

incremental gain of ECS over minimal screening (" $77 \%$ Intervention CF23 + SMA" in Table 2), showing that ECS averts an additional 200 births per 100,000 pregnancies ("77\% Intervention: Difference" in Table 2). Results were also modeled for a population less inclined toward reproductive intervention (Table 2: "50\% Intervention").

We combined estimates of incidence and disease cost to measure the differences in medical spending associated with ECS panel conditions. Because different stakeholders may prioritize different time horizons, we examined three periods: lifetime, years $0-3$, and years $0-1$, where year 0 is birth. This modeling focused entirely on disease treatment costs and did not take into account the cost of screening or reproductive care (discussed later).

In the population impact (i.e., $100 \%$ intervention) scenario, the avoided costs averaged over all births-the minority affected plus the majority unaffected-were approximately $\$ 3200$ in lifetime costs, $\$ 410$ in years $0-3$ costs, and $\$ 130$ in years $0-1$ costs (Table 2 ). Among only affected births, the average lifetime cost per affected birth was $\$ 1,100,000$. Based on the empirical cohort wherein $77 \%$ of ARCs intervened to avoid an affected birth ${ }^{18}$ and using CF 23 + SMA as a baseline, ECS showed incremental cost savings when averaged over all births of approximately $\$ 1800$ over a lifetime, $\$ 210$ in years $0-3$, and $\$ 70$ in years $0-1$.

We also modeled the cost of reproductive care for those ARCs who intervene to avoid an affected pregnancy (Table 2: "Intervention costs accrued"). Because ARCs are relatively rare (1.2\% as modeled herein) and some choose not to pursue further intervention, the per-couple contribution of these costs is low (\$160) when compared with the disease treatment costs on the three-year or lifetime horizons ( $\$ 410$ and $\$ 3200$, respectively).

\section{Cost-effectiveness of ECS}

We examined the cost-effectiveness of preconception ECS (Fig. 2a) by treating the ECS price as a variable and plotting the incremental cost-effectiveness ratio (ICER) with or without modeled cost savings (for years 0-3) due to averted disease. Because insurer coverage for IVF expenses is highly variable, the modeling in Fig. 2 excludes such expenses (Fig. S1 shows the analysis including IVF expenses). For comparison, cost-effectiveness values obtained for hereditary cancer screening are also shown. ${ }^{10,35-37}$

Compared with no screening, "77\% Intervention ECS" showed a cost-effectiveness near the common benchmark value of $\$ 50,000$ per life-year ${ }^{38}$ (solid and hatched magenta bars in Fig. 2a), particularly when cost savings due to avoided disease are included in the analysis (solid magenta bars in Fig. 2a). To illustrate this calculation, consider a hypothetical example with rounded numbers: if ECS were priced at $\$ 1000$, saved 0.01 life-years on average, and we accounted for $\$ 400$ in cost savings, the ICER would be $(\$ 1000-\$ 400) / 0.01=$ $\$ 60,000$ per life-year. Further, when costs are evaluated on a lifetime horizon (rather than 3-year horizon), ECS is costsaving at prices up to $\$ 2500$ because the net averted costs are greater than the price. Because some populations may intervene at lower rates, we also modeled the ICER of ECS versus no screening for all possible choices of intervention rate (Fig. 2b).

Compared with CF 23 + SMA screening, "77\% Intervention ECS" showed greater cost-effectiveness (Fig. 2a; lime). The price of CF23 + SMA screening (\$693.60) was estimated as the median price paid for CF23 + SMA screening (CPT codes 81220,81401 ) across US commercial laboratories, as reported under the Protecting Access to Medicare Act (PAMA). ${ }^{39}$ The improved cost-effectiveness of ECS in this comparison arose for two reasons. First, the price of CF23 + SMA screening was large when compared with the ECS prices examined. Second, the majority of ECS clinical impact (affected births averted and life-years gained) stemmed from panel conditions beyond CF23 and SMA. Together, the ICER of ECS relative to CF23 + SMA was high because the improved clinical outcomes from ECS were achieved for a smaller marginal cost 
a

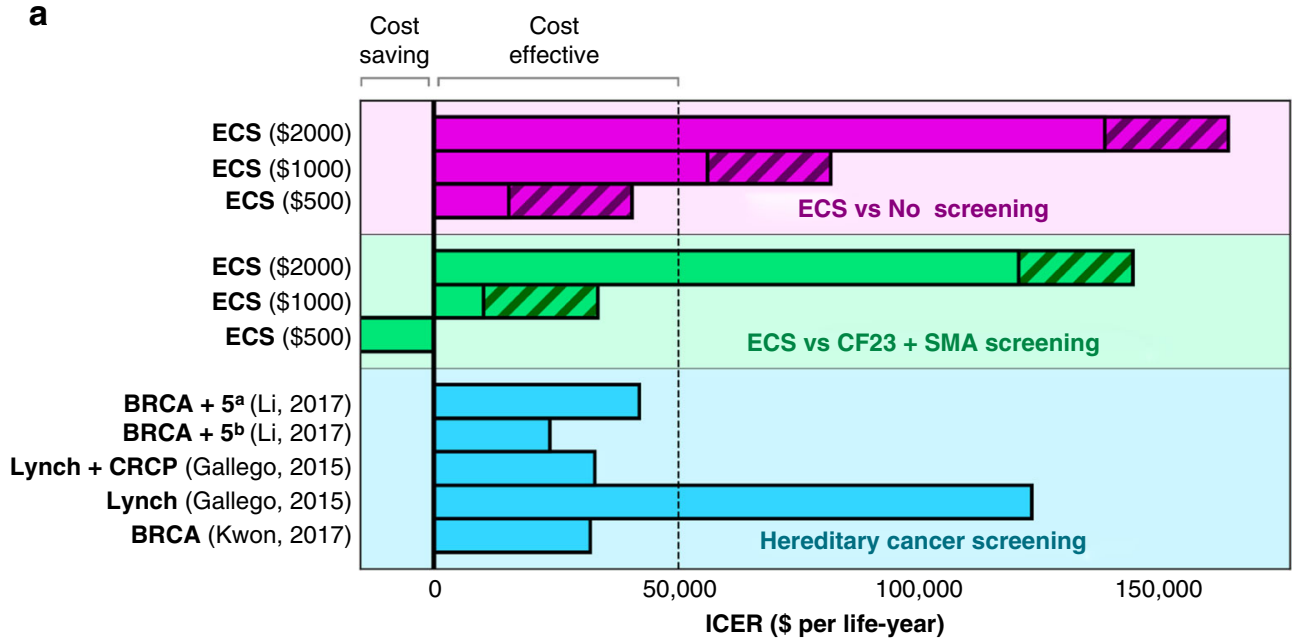

b

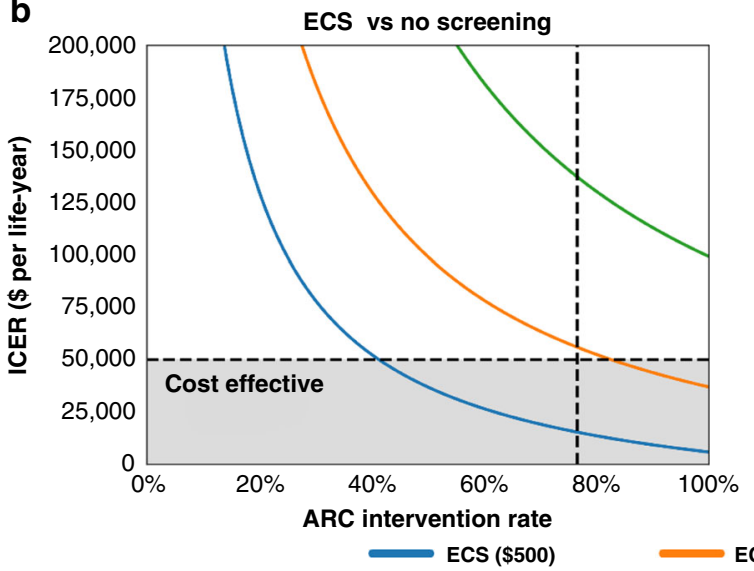

C ECS vs CF23 + SMA screening

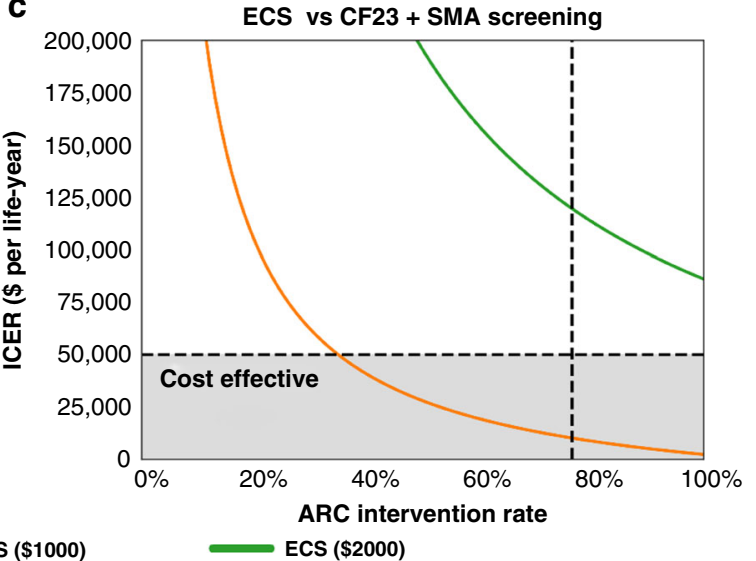

Fig. 2 Expanded carrier screening (ECS) cost-effectiveness. (a). The incremental cost-effectiveness ratio (ICER) of "77\% Intervention ECS" is compared with no screening (magenta) and cystic fibrosis/spinal muscular atrophy (CF23 + SMA) screening (green). Life-years gained are used as clinical outcomes of interest. ECS price is shown in parentheses. Solid bars indicate the ICER modeled with 3-year cost savings subtracted, while hatched bars indicate the ICER without such deduction (i.e., only accounting for screening cost). As a comparison, ICER values are shown for several inherited cancer interventions (blue). The common $\$ 50,000$ per life-year cost-effectiveness threshold is shown as dashed lines; note, however, that thresholds as high as $\$ 100,000$ have been proposed. ${ }^{42}$ Superscripts ${ }^{a}$ and ${ }^{b}$ refer to multiple ICER estimates from the same study. In the green section, the third bar ("ECS [\$500]") is cost saving because after subtracting the price of CF23 + SMA screening (\$693.60), the cost per life-year is negative. The plot is truncated because negative ICER results are typically not interpreted quantitatively, as one alternative is superior to the other in terms of both cost and life-years saved. See Fig. S1 for a similar analysis that also includes in vitro fertilization (IVF) expenses. Quantitative values are given in Table S10. (b, c) Modeled cost-effectiveness-including the impact of three-year cost savings-is shown across a range of at-risk couple (ARC) intervention rates. (b) Cost-effectiveness versus no screening. (c) Costeffectiveness versus CF + SMA screening; note that at $\$ 500$, the intervention is cost saving and, therefore, omitted from the plot because of CF + SMA pricing. Vertical dashed lines in (b, c) indicate the $77 \%$ intervention rate; horizontal lines indicate the $\$ 50,000$ per life-year cost-effectiveness level.

increase than the ICER calculations that used no screening as a baseline. At low price points (i.e., near CF23 + SMA pricing of \$693.60), ECS is highly cost-effective and incrementally cost-saving-that is, the additional averted disease costs outweigh the incremental price difference between ECS and CF23 + SMA screening (Fig. 2a). Because some populations may intervene at lower rates, we also modeled the ICER of ECS versus CF23 + SMA screening for all possible choices of intervention rate (Fig. 2c).

\section{Sensitivity analysis to assess robustness}

We assessed model robustness to both single-parameter variation (one-way sensitivity analysis) and multivariate parameter uncertainty (probabilistic sensitivity analysis). In one-way sensitivity analysis (Fig. 3; bottom), we probed several model parameters: (1) the population ARC frequency, (2) the fraction of ARCs who alter their reproductive behavior, (3) the fraction of CF risk attributable to the 23 ACMG-recommend variants, and (4) the assumed lifetime cost value for conditions without a published value. As expected, the model was most sensitive to the population ARC frequency, which could vary greatly when applying ECS to high-risk, intermediate-risk, and low-risk ethnicities. The model showed less sensitivity to the other model parameters.

The probabilistic sensitivity analysis largely reflected the range of values observed in the one-way sensitivity analysis when scaling the population ARC frequency (Fig. 3; top); this correspondence was unsurprising given the dominant 

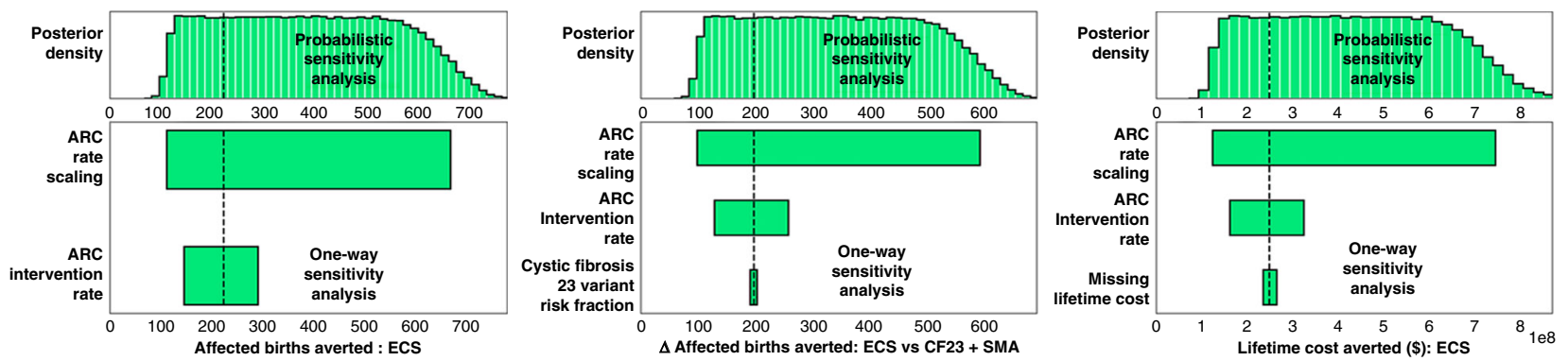

Fig. 3 Sensitivity analysis. Bottom: One-way sensitivity analysis tornado plot shows sensitivity to model parameters for affected births averted by expanded carrier screening (ECS), difference in affected births averted between ECS and cystic fibrosis/spinal muscular atrophy (CF23 + SMA) screening, and lifetime costs averted by ECS. Top: Probabilistic sensitivity analysis shows the sensitivity of affected births averted due to multivariate parameter uncertainty. Results are per 100,000 couples. ARC at-risk couple.

contribution that this parameter showed in the one-way analyses. The probabilistic sensitivity analysis used the prior distributions enumerated in Table S2 and suggested that, for every 100,000 births, the median number of averted affected births was 384 (95\% credible interval: 123-678).

\section{DISCUSSION}

Here, a decision tree model integrating incidence, clinical impact, and cost revealed several new findings about ECS. First, the cost of disease is large, even when averaged over a population of affected and unaffected births. Second, the clinical benefits of screening (affected births averted and lifeyears gained) are largely attributable to diseases beyond CF23 + SMA. Third, the cost-effectiveness of ECS is estimated to be favorable (at a threshold of $\$ 50,000$ per life-year), particularly when averted disease costs are considered and when compared against CF23 + SMA screening. We also observed that high-cost diseases tend to have high-priced, recently approved drugs (e.g., Spinraza) or long life expectancy with treatment (e.g., CF), suggesting that financial impact of Mendelian diseases may increase as new orphan drugs obtain approval.

Modeling carrier screening is complicated because of the choices involved in what to model, including whether screening occurs before or after conception, which interventions are chosen among different cohorts, and how well screening results correspond to affected births. While more complex decision tree models have attempted to capture several of these variations, ${ }^{7}$ they required many uncertain parameters (e.g., rates of specific reproductive choices) and emphasized individual carrier status rather than the more clinically relevant ARC status. In this work, we pursued the following approach: (1) model a simple ECS workflow that captures the key clinical management steps of carrier screening, and (2) account for simplifying assumptions by assessing uncertainty in the model's conclusions with sensitivity analysis. In this model of preconception ECS, we also assume that each reproductive intervention leads to $100 \%$ avoidance of disease (via, e.g., replacement of an affected birth by a healthy one); this assumption, which is favorable to ECS, is worth revisiting in future work that considers ECS outside of the preconception period.
While many couples undergo prenatal ECS, preconception screening was the focus of our model because it confers greater patient autonomy. ${ }^{3}$ The fewer options available to prenatal couples have observable impact on reproductive decision-making, with a $\sim 37 \%$ (rather than $\sim 77 \%$ ) rate of reproductive interventions at that stage. ${ }^{18}$ Therefore, although full characterization of the health economics of prenatal screening is outside the scope of this study, we expect the economic impact of prenatal screening to be slightly below that of the $50 \%$ model (Table 2; the impact on costeffectiveness of variable intervention rate is in Fig. $\mathbf{2 b}, \mathbf{c}$ ).

Expenditures associated with reproductive interventions such as IVF and PGT-M may accrue to different parties, depending on the couple's insurance. With the understanding that stakeholders assess these costs differently, we separately accounted for disease costs and reproductive intervention costs in Table 2 to empower future stakeholder-tailored analyses.

The ARC intervention rate used herein is based on survey responses of ARCs. ${ }^{18}$ Although that study was among the largest studies of actions taken by ECS ARCs, the survey population was, when compared with a general US population, overenriched for Ashkenazi Jewish persons and underenriched for African Americans. Stakeholders applying these results to specific regions or populations may wish to model the intervention rate appropriate for their target cohort; such reanalysis can be achieved using the data in Table 2, Fig. 2, and Fig. 3. Future studies may be warranted to better understand the reproductive behaviors of patient groups not currently receiving ECS.

We note three challenges in modeling birth incidence of ECS conditions. First, our modeling counts carrier frequencies from ECS results and models incidence via known (autosomal recessive or X-linked) inheritance. This approach leads to reasonable but imperfect concordance with observational approaches; discrepancies arise from statistical uncertainty (in both modeled and observed rates), demographics (ethnicity and intermarriage rate), variable disease penetrance, and elevated cohort risk not fully addressed by our cohort selection process (see Table S8). Second, we assumed perfect sensitivity and specificity for screened diseases. The assumption of perfect test accuracy-which we submit is justified 
given the $>99 \%$ analytical sensitivity and specificity reported in the validation of the 176 -condition ECS panel ${ }^{23}$-means that an affected birth can only result from either not screening for a particular disease or an ARC not pursuing intervention. Clinical specificity is expected to be high, ${ }^{28}$ and even if clinical sensitivity were $<100 \%$, the results of our cost-effectiveness analysis would not be impacted because the clinical benefit measurements (i.e., the denominator in the cost-effectiveness equation) rely only on variants known to be pathogenic and are not affected by the undetected or incorrectly classified pathogenic variants that decrease clinical sensitivity. Third, though miscarriage can be common for certain diseases (e.g., Smith-Lemli-Opitz syndrome ${ }^{40}$ ) and may impact cost estimates, we expect this impact to be minor because few diseases and variants yield higher miscarriage rates.

Although not the focus of this study, it is important to note that ECS provides benefits beyond the reduction of affected births and the increase in expected life-years. These include early education for conditions associated with intellectual disability (e.g., in fragile $\mathrm{X}$ syndrome ${ }^{41}$ ), early detection of impairment that can lead to intervention or treatment (e.g., cochlear implants to treat GJB2-related deafness), communication of risk to family, and faster diagnoses of rare disorders that could otherwise require a lengthy diagnostic odyssey. Importantly, many of these benefits accrue to couples who choose not to avoid an affected pregnancy. Though accounting for them is difficult in a quantitative costeffectiveness framework, they are benefits nonetheless.

\section{ELECTRONIC SUPPLEMENTARY MATERIAL}

The online version of this article (https://doi.org/10.1038/s41436019-0455-8) contains supplementary material, which is available to authorized users.

\section{DISCLOSURE}

All authors are employees of Myriad Women's Health (MWH).

Publisher's note: Springer Nature remains neutral with regard to jurisdictional claims in published maps and institutional affiliations.

\section{REFERENCES}

1. Langlois S, Benn P, Wilkins-Haug L. Current controversies in prenatal diagnosis 4: pre-conception expanded carrier screening should replace all current prenatal screening for specific single gene disorders. Prenat Diagn. 2015;35:23-28.

2. Kingsmore $\mathrm{S}$. Comprehensive carrier screening and molecular diagnostic testing for recessive childhood diseases. PLoS Curr. 2012. https://doi.org/ 10.1371/4f9877ab8ffa9. Accessed 11 February 2019.

3. Committee opinion no. 690 summary: carrier screening in the age of genomic medicine. Obstet Gynecol. 2017;129:595-596.

4. Committee opinion no. 691 summary: carrier screening for genetic conditions. Obstet Gynecol. 2017;129:597-599.

5. Grody WW, et al. Laboratory standards and guidelines for populationbased cystic fibrosis carrier screening. Genet Med. 2001;3:149-154.

6. Lazarin GA, et al. An empirical estimate of carrier frequencies for $400+$ causal Mendelian variants: results from an ethnically diverse clinical sample of 23,453 individuals. Genet Med. 2013;15:178-186.

7. Azimi $M$, et al. Carrier screening by next-generation sequencing: health benefits and cost effectiveness. Mol Genet Genomic Med. 2016;4:292-302.
8. AMA. AMA CPT Editorial Summary. 2017. https://www.ama-assn.org/ sites/ama-assn.org/files/corp/media-browser/public/physicians/cpt/june2017-summary-panel-actions.pdf. Accessed 11 February 2019.

9. Briggs $\mathrm{AH}$, et al. Model parameter estimation and uncertainty: a report of the ISPOR-SMDM Modeling Good Research Practices Task Force-6. Value Health. 2012;15:835-842.

10. Li $Y$, et al. A multigene test could cost-effectively help extend life expectancy for women at risk of hereditary breast cancer. Value Health. 2017;20:547-555.

11. Spackman E, Hinde $S$, Bojke $L$, et al. Using cost-effectiveness analysis to quantify the value of genomic-based diagnostic tests: recommendations for practice and research. Genet Test Mol Biomarkers. 2017;21:705-716.

12. Little $S E$, et al. The cost-effectiveness of prenatal screening for spinal muscular atrophy. Am J Obstet Gynecol. 2010;202:253.e1-7.

13. Musci TJ, Caughey $A B$. Cost-effectiveness analysis of prenatal population-based fragile $X$ carrier screening. Am J Obstet Gynecol. 2005;192:1905-1912. discussion 1912-5

14. Lieu TA, Watson SE, Washington AE. The cost-effectiveness of prenatal carrier screening for cystic fibrosis. Obstet Gynecol. 1994;84:903-912.

15. Norman R, van Gool K, Hall J, et al. Cost-effectiveness of carrier screening for cystic fibrosis in Australia. J Cyst Fibros. 2012;11:281-287.

16. Schultz, NM, Daines, CL \& Malone, DC A Cost-Effectiveness Analysis of Ivacaftor (Kalydeco?) for Treatment of Cystic Fibrosis. in B102. NOVEL APPROACHES TO UNDERSTAND AND IMPROVE HEALTH OUTCOMES A3713-A3713 (American Thoracic Society, 2015). American Journal of Respiratory and Critical Care Medicine 2015;191:A3713. https://www.atsjournals.org/doi/abs/ 10.1164/ajrccm-conference.2015.191.1_MeetingAbstracts.A3713. Accessed 11 February 2019.

17. National Center for Pharmacoeconomics. Cost-effectiveness of Nusinersen (Spinraza) for the treatment of $5 q$ spinal muscular atrophy (SMA). (2017). http://www.ncpe.ie/wp-content/uploads/2017/08/ Summary-Nusinersen.pdf. Accessed 11 February 2019.

18. Johansen Taber, KA, Beauchamp KA, Lazarin G, et al. Clinical utility of expanded carrier screening: results-guided actionability and outcomes. Genet Med. 2018; https://www.nature.com/articles/s41436-018-0321-0 [Epub ahead of print]. Accessed 11 February 2019.

19. Ghiossi CE, Goldberg JD, Haque IS, et al. Clinical utility of expanded carrier screening: reproductive behaviors of at-risk couples. J Genet Couns. 2018;27:616-625.

20. Archibald $A D$, et al. Reproductive genetic carrier screening for cystic fibrosis, fragile $X$ syndrome, and spinal muscular atrophy in Australia: outcomes of 12,000 tests. Genet Med. 2018;20:513-523.

21. Lazarin GA, et al. Systematic classification of disease severity for evaluation of expanded carrier screening panels. PLoS ONE. 2014;9:e114391.

22. Markus AR, Andres E, West KD, et al. Medicaid covered births, 2008 through 2010, in the context of the implementation of health reform. Womens Health Issues. 2013;23:e273-80.

23. Hogan GJ, et al. Validation of an expanded carrier screen that optimizes sensitivity via full-exon sequencing and panel-wide copy number variant identification. Clin Chem. 2018;64:1063-1073.

24. Dunn A, Grosse SD, Zuvekas SH. Adjusting health expenditures for inflation: a review of measures for health services research in the United States. Health Serv Res. 2018;53:175-196.

25. St. Louis Federal Reserve. FRED: personal consumption expenditures: services: health care. 2018. https://fred.stlouisfed.org/ series/DHLCRG3Q086SBEA. Accessed 11 February 2019.

26. Weinstein MC, Siegel JE, Gold MR, et al. Recommendations of the panel on cost-effectiveness in health and medicine. JAMA. 1996;276:1253-1258.

27. Haque IS, et al. Modeled fetal risk of genetic diseases identified by expanded carrier screening. JAMA. 2016;316:734-742.

28. Beauchamp KA, et al. Systematic design and comparison of expanded carrier screening panels. Genet Med. 2018;20:55-63.

29. US Census. American FactFinder. Available at: http://factfinder.census. gov/faces/nav/jsf/pages/index.xhtml. Accessed 11 February 2019.

30. Feng LB, Grosse SD, Green RF, et al. Precision medicine in action: the impact of ivacaftor on cystic fibrosis-related hospitalizations. Health Aff (Millwood). 2018;37:773-779.

31. Sacco P, Capkun-Niggli G, Zhang $X$, Jose R. The economic burden of fragile $X$ syndrome: healthcare resource utilization in the United States. Am Health Drug Benefits. 2013;6:73-83.

32. Adamson, DM, Chang, $S \&$ Hansen, LG Health research data for the real world: the MarketScan databases. New York: Thompson Healthcare (2008). 
33. Reeves $S$, et al. Identifying sickle cell disease cases using administrative claims. Acad Pediatr. 2014;14:S61-67.

34. Watson MS, et al. Cystic fibrosis population carrier screening: 2004 revision of American College of Medical Genetics mutation panel. Genet Med. 2004;6:387-391.

35. Kaldate $R$, Huston $A, M c C o y ~ H$, et al. Cost effectiveness analysis of genetic testing for breast and ovarian cancer susceptibility genes: BRCA1 and BRCA2. Breast J. 2014;20:325-326.

36. Gallego CJ, et al. Next-generation sequencing panels for the diagnosis of colorectal cancer and polyposis syndromes: a cost-effectiveness analysis. Clin Oncol. 2015;33:2084-2091.

37. Kwon JS, Daniels MS, Sun CC, Lu KH. Preventing future cancers by testing women with ovarian cancer for BRCA mutations. J Clin Oncol. 2010;28:675-682.

38. Owens DK. Interpretation of cost-effectiveness analyses. J Gen Intern Med. 1998;13:716-717.

39. PAMA 2018. CY 2018 - Preliminary Private Payor Rate-Based CLFS Payment Rates and Analytics. https://www.cms.gov/Medicare/MedicareFee-for-Service-Payment/ClinicalLabFeeSched/Downloads/CY2018-CLFSPayment-Rates-Analytics.zip. Accessed 11 February 2019.

40. Lazarin GA, Haque IS, Evans EA, Goldberg JD. Smith-Lemli-Opitz syndrome carrier frequency and estimates of in utero mortality rates. Prenat Diagn. 2017;37:350-355.

41. Hagerman RJ, et al. Advances in the treatment of fragile $X$ syndrome. Pediatrics. 2009;123:378-390.
42. Berry SR, et al. Continental divide? The attitudes of US and Canadian oncologists on the costs, cost-effectiveness, and health policies associated with new cancer drugs. J Clin Oncol. 2010;28:4149-4153.

(1) (2) Open Access This article is licensed under a Creative Commons (c) Attribution-NonCommercial-ShareAlike 4.0 International License, which permits any non-commercial use, sharing, adaptation, distribution and reproduction in any medium or format, as long as you give appropriate credit to the original author(s) and the source, provide a link to the Creative Commons license, and indicate if changes were made. If you remix, transform, or build upon this article or a part thereof, you must distribute your contributions under the same license as the original. The images or other third party material in this article are included in the article's Creative Commons license, unless indicated otherwise in a credit line to the material. If material is not included in the article's Creative Commons license and your intended use is not permitted by statutory regulation or exceeds the permitted use, you will need to obtain permission directly from the copyright holder. To view a copy of this license, visit http://creativecommons.org/licenses/by-nc-sa/4.0/.

(c) The Author(s) 2019 